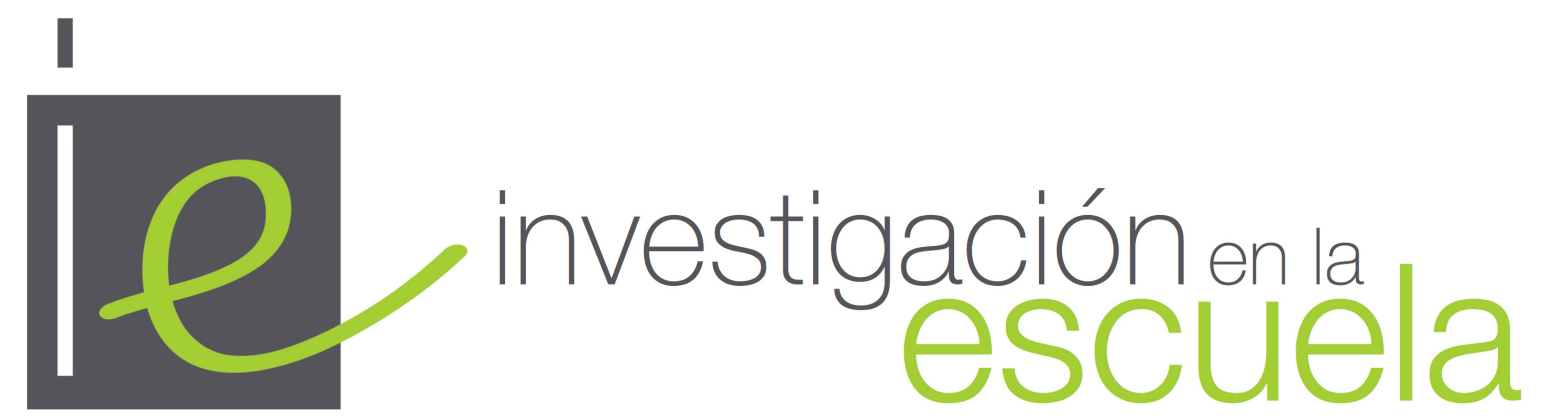

Revista internacional de investigación e innovación educativa

\title{
Investigar en educación desde una posición de no saber: dar cuenta de los tránsitos de una investigación en torno a cómo aprende el profesorado de secundaria
}

\author{
Fernando Hernández-Hernández \\ Universidad de Barcelona \\ España
}

Citación: Hernández-Hernández, F. (2019). Investigar en educación desde una posición de no saber: dar cuenta de los tránsitos de una investigación en torno a cómo aprende el profesorado de secundaria. Investigación en la Escuela, 99, 1-14. doi: http://dx.doi.org/10.12795/IE.2019.i99.01

Resumen: ¿Cómo se puede afrontar una investigación que no siga un camino predefinido ni aplique unos métodos preestablecidos, sino que se abra al diálogo con lo que emerge en el encuentro con las experiencias de los colaboradores y del investigador? ¿Qué efectos tiene en el investigador un proceso de investigación que se ve alterado por lo imprevisto y por ponerle en una posición de no saber? ¿Qué sucede cuando se quiebra el sentido hegemónico de investigar, que parte de diseñar una situación que permita generar unos datos, para luego analizarlos y dar cuenta de sus posibles sentidos de acuerdo con las decisiones previamente establecidas? Estas son algunas de las preguntas presentes en este artículo en el que se da cuenta de los tránsitos de un investigador por un proyecto que indaga cómo el profesorado de secundaria aprende y de cómo este recorrido afecta al investigador. Esta noción de afectar hace que el proceso de indagación se configure como un recorrido relacional donde se está atento, no a lo que se espera encontrar, sino a lo que (nos) va aconteciendo en un proceso que no es lineal, sino repleto de bifurcaciones, dudas y lugares de no 
saber. Proceso que permite afrontar un sentido de investigación que revisa y cuestiona algunos fundamentos y prácticas sobre lo que se asume de manera normalizada como investigar.

Palabras clave: "Investigación postcualitativa"; "indagación"; "devenir"; "aprender"; "profesorado de secundaria"; "Deleuze"; "afectos"; "no saber"

Research in education from a position of not knowing: reporting the transits of an investigation into how secondary school teachers learn

Abstract: How can we face research that does not follow a predefined path or apply preset methods, but opens up to dialogue with what emerges in the encounter with the experiences of the collaborators and the researcher? What is the effect of a research process when is altered by the unforeseen and by putting the researcher in a position of unknowing? What happens when is broken the hegemonic meaning of the research which starts from designing a situation that allows for the generation of data in order to analyze them and account for their possible meanings in accordance with previously established decisions? These are some of the questions present in this article in which the transits of a researcher through a project that investigates how secondary school teachers learn and how this journey affects the researcher are accounted for. This affecting makes the inquiry process configure itself as a relational journey where one is attentive not to what one expects to find, but to what (us) is happening in a process that is not linear, but full of bifurcations, doubts and places of not knowing. A process that allows to confront one another sense of research, that revises and questions some foundations and practices on what is assumed in a normalized way as research. Key words: "Post-qualitative research"; "inquiry"; "becoming"; "learning"; "secondary school teachers"; "Deleuze"; "affects"; "unknown".

La recherche en éducation à partir d'une position d'ignorance : rapporter les passages d'une enquête sur la façon dont les enseignants du secondaire apprennent

Resumè: Comment faire face à une recherche qui ne suit pas un parcours prédéfini ou n'applique pas des méthodes préétablies, mais s'ouvre au dialogue avec ce qui émerge dans la rencontre avec les expériences des collaborateurs et du chercheur? Quel est l'effet d'un processus de recherche lorsqu'il est modifié par l'imprévu et en mettant le chercheur dans une position d'inconnu ? Que se passe-t-il quand est rompu le sens hégémonique de la recherche qui commence par la conception d'une situation permettant de générer des données afin de les analyser et de rendre compte de leurs significations possibles conformément à des décisions préalablement établies ? Telles sont quelquesunes des questions posées dans cet article où l'on rend compte des transits d'un chercheur dans le cadre d'un projet qui examine comment les enseignants du secondaire apprennent et comment ce parcours affecte le chercheur. Cet affectant fait que le processus d'enquête se configure comme un parcours relationnel où l'on est attentif non pas à ce que l'on s'attend à trouver, mais à ce qui (nous) se passe dans un processus qui n'est pas linéaire, mais plein de bifurcations, de doutes et de lieux d'incompréhension. Un processus qui permet de se confronter au sens de la recherche, qui révise et remet en question certains fondements et pratiques sur ce qui est supposé être de la recherche de façon normalisée.

Mots-clés: "Recherche post-qualitative"; "enquête"; "devenir"; "apprendre"; "enseignants du secondaire"; "Deleuze"; "affections"; "inconnu". 


\section{Situar el interés en torno a cómo aprenden los docentes}

Podemos ser invasoras de espacios en los círculos académicos; también podemos ser invasoras de espacios en la teoría, solo por citar textos inconvenientes o por hacer preguntas inconvenientes (Ahmed, 2018, p. 24).

Cómo aprenden los docentes es una cuestión que no me es ajena y que desde hace años me acompaña ${ }^{1}$. Se hizo patente en el marco de la reforma que promovió la Ley Orgánica General del Sistema Educativo (LOGSE) a partir de 1990, con los docentes a quienes se trataba de transmitir otra fundamentación que guiara sus prácticas. Una formación basada en la idea de aprender por recepción y repetición para luego reproducirlo. Pero sabía entonces, por lo que había ocurrido en los años 70 en la reforma de la enseñanza de las ciencias en Estados Unidos, que los docentes no transforman las ideas que orientan su práctica cuando se enfrentan a una propuesta que las cuestiona. Lo que hace necesario, como señalan Darling-Hammond y Richardson (2009), que para diseñar experiencias de desarrollo profesional y propuestas de cambio hay que tener en cuenta cómo los docentes aprenden. Esta idea, que entonces fue para mí una intuición relacionada con las prácticas de formación en las que participaba, me llevó a publicar un artículo titulado Cómo aprenden los docentes (Hernández, 1996-1997). Desde él respondía a la inquietud que entonces despertó en mí darme cuenta de que tanto en las investigaciones promovidas por el Centro de Investigación y Documentación Educativa (CIDE) en el periodo 1983-1993, como en las revisiones realizadas por Marcelo (1995) y Barquín (1995), los estudios sobre el profesorado se orientaban a sus características sociológicas, al pensamiento docente y al estrés escolar.

Desde entonces he seguido con interés la investigación sobre el aprender de los docentes, sobre todo la más extendida, y que se fundamenta en las dimensiones del modelo de conocimiento profesional de Shulman y Shulman (2004): Pedagogical Content Knowledge (PCK). Modelo que incluye el contenido disciplinar, el didáctico, el pedagógico-práctico y el contextual, junto con las competencias transversales para el profesorado experimentado. Si bien esta ha sido una línea dominante, Opfer y Pedder (2011), en la revisión que realizan sobre el tema en la Review of Educational Research, señalan que para comprender cómo se configura el aprendizaje docente, es necesario situarlo en relación con cuatro subsistemas: el docente, la escuela, la actividad de aprendizaje y las experiencias de aprendizaje del profesorado fuera de su práctica profesional. Siendo este último subsistema el que ha sido menos estudiado.

Fue siguiendo este hilo conductor, presentado aquí de forma somera, lo que nos llevó a los grupos de investigación Esbrina y Elkarrikertuz ${ }^{2}$ a indagar cómo el profesorado aprende dentro y fuera de las instituciones educativas. Pero, al igual que nos sucedió cuando nos acercamos al tema de cómo se aprende a ser sujeto en la escuela primaria (Hernández-Hernández, 2010), el desafío que se nos planteó fue cómo investigar sobre la experiencia del aprender, que es una noción resbaladiza (Fendler, 2015) y que se escapa entre los dedos cuando te acercas a ella, si se pretende convertirla en datos.

Se hizo patente esta dificultad mientras escribía el informe de medio tiempo sobre el proyecto Apren-do ${ }^{3}$ al reflexionar sobre cómo la segunda generación de la Teoría Fundamentada

\footnotetext{
${ }^{1}$ Este artículo está escrito en primera persona porque se centra en los cambios que afectan al autor cuando participa en una investigación, en la que la pregunta de partida cambia cuando se entra en contacto con los docentes.

2 https://esbrina.eu/es/inicio/; https://www.ehu.eus/es/web/elkarrikertuz/home

3 APREN-DO: Cómo aprenden los docentes: implicaciones educativas y retos para afrontar el cambio social. Ministerio de Economía y Competitividad / EDU2015-70912-C2-1-R
} 
(Charmaz, 2000, 2006), que guiaba este estudio, suponía una limitación para dar cuenta de la complejidad de la experiencia de aprender. Seguir esta perspectiva hacía que las cartografías de las trayectorias de aprendizaje, que nos regalaron los docentes de secundaria a los que invitamos a participar en la investigación, tenían que ser consideradas como evidencias para ser analizadas desmenuzadas- y hacerles decir, mediante un proceso analítico de categorización, aquello que nos permitiera confirmar o no, nuestras posiciones iniciales. Además de responder a las preguntas hechas antes de encontrarnos con los docentes y sus experiencias (y las nuestras) en el proceso de generar las cartografías de aprendizaje.

En la propuesta de la investigación, las cartografías se configuraban como un método desencadenante que tenía que permitir a los docentes hablar sobre lo que para ellos era aprender, dónde aprendían y cómo transitaban dentro y fuera de los institutos. Las cartografías eran una estrategia visual para llegar al texto, a la palabra.

Si las cartografías se nos presentaban con algo a desenredar, nosotros lo haríamos analizando el contenido de las mismas, aislando sus unidades de significado y poniéndolas en relación con lo que el profesorado había dicho (el texto) frente a ellas (Guattari, 2000; Guattari y Rolnik, 2006; Masny, 2013; Ulmer y Koro-Ljungberg, 2015). De esta manera, transformaríamos la experiencia en datos. Esto ocurriría al poner los textos en un programa informático, o al realizar de forma manual tablas, en las que iríamos realizando, de manera escalonada, lo que habíamos escrito inicialmente en el proyecto.

Pero esto no lo hicimos, no porque no tuviéramos capacidad y recursos para hacerlo, sino porque desde el momento en el que entramos en contacto con nuestras propias cartografías (Canales, Padilla-Petry y Gutiérrez, 2019), nos dimos cuenta de que lo que aparecía en el proceso de cartografiar perdía su riqueza y complejidad si lo reducíamos a datos. Esta consciencia hizo que, en varios periodos de la investigación, nos sintiéramos desorientados, pues no teníamos la certeza sobre cómo transitar por un camino que no redujera la experiencia a lo que nosotros, los investigadores, queríamos encontrar. Nos dimos cuenta que necesitábamos otro marco ontoespistemológico, metodológico y ético, para dar salida a los desafíos que nos planteaba la riqueza de los planos de sentido, desde los que los docentes proyectaban su aprender.

En este punto, algo estaba cambiando en nosotros sobre lo que podía ser investigar, pero no sabíamos dónde nos iba a llevar. Comenzamos entonces a explorar no cómo analizar las cartografías, sino cómo tomarlas como espacios de encuentro que nos posibilitaban pensar a través de ellas sobre conceptos vinculados al aprender: los afectos, la corporealidad, el espacio y el tiempo, las intraacciones... Esto suponía prestar atención a los movimientos, a los enredos, a los gestos, a los 'planos de realidad' (Jackson y Mazzei, 2012) que se entrecruzaban entre las cartografías y los relatos que los docentes construían en su pensar sobre su aprender. Suponía, además, desplazar la pregunta 'qué es el aprender para los docentes' a 'cómo los docentes dicen que aprenden'. Y no en detenernos en lo que 'dicen' las cartografías sino en lo que 'hacen'.

De cómo se gestó este movimiento, de los efectos que tuvo en la investigación y, sobre todo, en mí como investigador, trata el contenido de las siguientes páginas. Aunque la investigación ha sido parte de un proceso grupal, en el que han participado investigadores de los dos grupos (Hernández y Hernández, Correa Gorospe, Aberazturi Apraiz y Sancho Gil, 2019) cada investigadora ha realizado un recorrido diferente. Dar cuenta de las relaciones entre esos recorridos podría ser el tema de otro artículo. Ahora me detengo en cómo me ha afectado para pensar desde otro lugar la investigación educativa. 


\section{Fundamentación: Otro sentido de lo que puede significar investigar en educación}

Se puede establecer un paralelismo entre cómo se entiende la docencia y la investigación. En cómo se asume ser docente e investigador. En esta relación hay una tradición dominante en la investigación que establece que las preguntas, el recorrido, el análisis y, en cierta forma, los resultados, han de ser previstos y enunciados de antemano. Algo similar sucede en la docencia. Lo que se pretende que los estudiantes aprendan se establece de antemano, así como los pasos, medios y estrategias para conseguirlo. La evaluación del aprendizaje, como de los resultados de la investigación, se establece en cuanto a su conformidad (o no) con lo previamente establecido. Pero hay otra manera de concebir la docencia y la investigación, que responde a otra concepción ontoepistemológica, metodológica y ética. Una aproximación menos reconocida porque "intimida y cuestiona las creencias profundamente arraigadas sobre el mundo" de aquellos "que tienen debilidad por los libros -de modos de hacer investigación y docencia- que refuerzan lo que saben y encajan bien en una categoría predeterminada" (Hustvet, 2019, p. 117, parafraseado).

Una perspectiva de la investigación (y de la docencia) que, como señala Patti Lather (2013), busca apartarse de las ortodoxias y se posiciona a favor de una investigación (una docencia) que ilumina, critica y evalúa lo que se entiende por investigación (y docencia). También lo hace ante la que se coloca bajo el paraguas de la perspectiva cualitativa. Una de las características de esta propuesta disruptiva de lo normalizado es revertir la orientación de la investigación (de la docencia), desde la 'creación de resultados' a la de dar cuenta de los tránsitos, desvíos y tensiones que se relacionan con aquello que el camino de la investigación (el proceso de pensar y conocer) le ha permitido pensar a los investigadores (a los docentes) ${ }^{4}$.

Esta dualidad entre modos de concebir la investigación y la docencia la planteo con una finalidad pedagógica, en el sentido de visibilizar perspectivas que no dejan de lado que pueden darse cruces y vinculaciones entre ellas. Pero que permite rescatar la distinción y la relación entre "transcendencia" e "inmanencia" de la que hablan Deleuze y Guattari (2006), y que Atkinson (2011, 2018) ha llevado al aprender en la institución escolar. Estos dos conceptos nos ayudan a poner nombre a cómo nos vamos situando en la investigación, en la docencia y en el estudio del que aquí se da cuenta, sobre cómo aprenden los docentes. La trascendencia tiene que ver con lo normativo y el 'deber ser' y, por tanto, construye una imagen de pensamiento 'dogmática' a la hora de planear y presentar una investigación o configurar un proceso de aprender. Mientras que la inmanencia se relaciona con los espacios de reapropiación y de ruptura frente a ese 'deber ser'. Estos últimos producen nuevas imágenes de pensamiento (Deleuze y Guttary, 2015), que habitan dentro de marcos de flexibilidad.

Desde la aproximación de Atkinson, la trascendencia implica una relación 'con', mientras que la inmanencia indica una relación 'dentro' de. Este movimiento de inmanencia pone en juego la noción de "intra-acción” (Barad, 2012), pues se generan cruces entre la experiencia de las cartografías, los bagajes (de ellas y nosotros), los movimientos y los encuentros, la materialidad del espacio físico en el que las experiencias tienen lugar y los dispositivos que permiten hacer las cartografías y dejar testimonios en los relatos y memorias de los encuentros en fotos y vídeos. Todo eso favorece que el proceso de investigación no venga dado, sino que se articule de una manera fluida e imprevista desde estos entramados de relaciones de inmanencia.

\footnotetext{
4 Para una introducción a la genealogía del movimiento postcualitativo en investigación educativa se puede consultar Hernández-Hernández y Revelles Benavente, 2019).
} 
Dar cuenta del modo de acercarnos a los movimientos de los docentes en torno a sus trayectos sobre dónde y cómo aprenden, requiere entonces replantear el sentido de cómo se suele entender una aproximación metodológica y su relación con la fundamentación onto-epistemológica, metodológica y ética de la investigación. Desde una aproximación postcualitativa (Lather, 2013; Lather y St. Pierre, 2013; St. Pierre, 2011), no se trata de diseñar una situación que permita generar unos datos para luego analizarlos y dar cuenta de sus posibles sentidos de acuerdo con las decisiones previamente establecidas. Asumir una posición 'post' supone, según St. Pierre (2011, p. 615), “[...] a radical break with the humanist, modernist, imperialist, representationalist, ob $\neg$ jectivist, rationalist, epistemological, ontological, and methodological assumptions of Western Enlight $\neg$ enment thought and practice" 5 .

En este marco, la noción de 'devenir' (becoming) es clave porque nos posibilita plantear la investigación y el dar cuenta de ella como un proceso en movimiento, no como un lugar predefinido desde el que partir y al que llegar. Esto implica, como señala Semetsky (2006), que los sujetos de la investigación (docentes e investigadores) son sujetos en proceso, porque

"as becoming, (are) always placed between two multiplicities, yet one term does not become the other; the becoming is something between the two ... Therefore, becoming does not mean becoming the other, but becoming-other"' (Semetsky, 2006, p. 6).

Este devenir (becoming) supone siempre una relación. Es un movimiento que siempre es 'entre' (between) y que se refleja en nuestros encuentros con los otros y con la materialidad que corporeiza todas la relaciones, en la medida en que participamos en "a dynamic series of prehensional relations through which beings try to take account of each other, and such relations are underpinned by a composition of feelings and conceptual processes"7 (Atkinson, 2018, p. 129).

Estar 'dentro', 'entre' y en 'devenir' me lleva a asumir que el sentido de investigar que se adopta no persigue encontrar y desvelar lo que está 'ahí fuera', pues se reconoce que el afuera está enredado con la mirada de quien investiga. Esto hace que el proceso de indagación (inquiry) sea un recorrido relacional, donde se está atento no a lo que se espera encontrar, sino a lo que (nos) va aconteciendo en un proceso que no es lineal, sino repleto de bifurcaciones, dudas y lugares de no saber (Atkinson, 2015, 2018). Fue desde esta posición desde la que tratamos de preguntarnos, no lo que queríamos encontrar sino lo que nos permitía pensar, así como en los conceptos que emergían en el proceso cartográfico y que iluminaban los sentidos del aprender de los docentes.

Para dar cuenta de lo que ha supuesto este recorrido, el proceso de escritura ha estado atravesado por desplazamientos y movimientos no previstos. Lo que ha generado que la escritura forme parte del proceso de indagación. Esto nos libera, siguiendo la invitación de Richardson y St. Pierre (2005, p. 262), "from trying to write a single text in which everything is said at once to everyone [...] writing is validated as a method of knowing" ${ }^{8}$. Esta actitud ante la escritura es la que voy desplegando en este texto.

\footnotetext{
5 "[...] una ruptura radical con los supuestos humanistas, modernistas, imperialistas, representacionalistas, objetivistas, racionalistas, epistemológicos, ontológicos y metodológicos del pensamiento y la práctica de la Ilustración occidental" (Traducción del autor).

6 "como devenir, (son) siempre colocados entre dos multiplicidades, pero un término no se vuelve el otro; el devenir es algo entre los dos. Por lo tanto, llegar a ser no significa llegar a ser el otro, sino llegar a ser-otro" (Traducción del autor). 7 "una serie dinámica de relaciones aprehensionales a través de las cuales los seres tratan de tener en cuenta a los demás, y dichas relaciones se sustentan en una composición de sentimientos y procesos conceptuales" (Traducción del autor). 8 "de tratar de escribir un solo texto en el que todo se diga a la vez a todos [...] la escritura se valida como un método de saber" (Traducción del autor).
} 


\section{La metodología y los resultados de la investigación y cómo afectan al trayecto del investigador}

(El desafío está en) "diseñar configuraciones nuevas de lo visible, lo decible y lo pensable; y, por eso mismo, un paisaje nuevo de lo posible. Pero a condición de no anticipar su sentido ni su efecto" (Rancière, 2010, p.105).

La propuesta que elaboramos para la convocatoria del Ministerio daba cuenta de un recorrido deseado, desde una ontología y epistemología cualitativa realista, en la que la fundamentación y la metodología se presentaban como dos partes de lo que se presuponía sería un proceso coherente y estructurado. Sin embargo, desde el comienzo, cuando los investigadores llevamos a cabo, como he mencionado más arriba, un taller para realizar nuestras cartografías respondiendo a una premisa del grupo (Hernández-Hernández, Sancho-Gil y Domingo-Coscollola, 2018) de no pedir a los otros lo que nosotros no hacemos primero, nos dimos cuenta de que entrábamos en un terreno de no saber. Que las cartografías suponían entrecruzar imágenes, palabras y relatos que daban cuenta de recorridos biográficos, pero también de deseos que se articulaban entre lo inusual y lo no dicho. Todo ello, en un marco que nos colocaba ante la dificultad de afrontar un problema -el de cartografiar- en el que tanto nosotros, como luego los docentes, entrábamos en un territorio inexplorado: la narración visual.

Con este bagaje contactamos, -después de realizar un estudio piloto con dos docentes que nos anticipó la abertura de lo que nos íbamos a encontrar-, con tres profesoras con las que habíamos tenido contactos previos y a las que les propusimos participar en la investigación. Pero lo imprevisto irrumpió a través de la propuesta que ellas nos plantearon: “¿por qué en lugar de hacerlo de manera individual no venís a nuestro instituto y lo hacemos con todos los profesores que quieran participar?”. Esto era algo que no estaba previsto en la propuesta que habíamos elaborado, pero era consecuente con lo que Opfer y Pedder (2011) señalan de la relevancia que el aprender en grupo tiene en la vida de los docentes.

Desde esta invitación nos encontramos readaptando lo que habíamos pensado como un acercamiento individual para llevarlo a una relación de grupo. Esto suponía asumir que los movimientos de los docentes, aunque fuéramos varios investigadores los que nos encontrásemos con ellos, ponían en cuestión la fantasía del investigador omnisciente que recoge en sus observaciones -sin interferencias- lo que sucede a su alrededor. Ir a los centros y encontramos con un grupo de docentes, nos situaba ante una relación con múltiples focos, que demandaba estar abiertos a lo imprevisto y al no saber. Decidimos, después de pensar cómo encarar la nueva situación, 'ponernos en suspenso' y escribir a cada participante dándoles las gracias y avanzándoles lo que podíamos hacer juntos. Les remitimos un acuerdo ético en el que hacíamos un resumen del proyecto, explicitando lo que esperábamos y lo que les ofrecíamos. También les enviamos los dos ejemplos de cartografías que habían hecho un profesor y una profesora durante lo que habíamos considerado como un estudio 'piloto' para la investigación. Con ello, de alguna manera, pensábamos que la situación en la que nos encontraríamos ya estaría informada y los docentes en disposición de realizar lo que les proponíamos. Pero no sucedió del todo como lo habíamos pensado.

Fuimos a los tres centros y en cada uno nos encontramos con una situación diferente que no podíamos prever de antemano. Cuando llegamos al primero, el grupo (9 mujeres y 2 hombres) estaba afectado por la intervención de un padre en contra de una docente; esta situación les generaba un malestar que les hacía dudar acerca de si participar o no de la propuesta que les habíamos hecho. En el segundo, quienes participaron (5 mujeres y 2 hombres), mantenían vínculos entre ellos por compartir un proyecto de integración de lenguas y habían respondido a la invitación por el interés que les comunicó una de las participantes, quién había sido nuestro contacto inicial. En el tercero, 
vinieron 6 mujeres y 1 hombre. Una de ellas era la directora, con quien inicialmente habíamos conectado, y el resto compartía con ella su interés por la mejora del centro. Como se puede intuir, fueron tres situaciones que demandaba modos de relación diferenciados, no sólo con los grupos sino con cada docente durante la realización de las cartografías.

A los centros fuimos en dos ocasiones. Durante la primera, tuvo lugar lo que hemos descrito en los párrafos anteriores. En la segunda, cuatro meses después, compartimos lo que nos habían hecho pensar las cartografías y las conversaciones mantenidas, abriendo así un nuevo modo de relación en el que intercambiamos cómo a ellas y a nosotros nos había afectado la experiencia compartida.

En paralelo, 3 profesoras vinieron a la universidad para realizar sus cartografías. De esta manera, 24 mujeres y 6 hombres nos regalaron sus historias y su tiempo. La presencia de mujeres no es solo una indicación sociológica de la composición actual del profesorado, sino una pista que nos ayudó a comprender el sentido biográfico, ecológico y corporeizado del aprender.

Este recorrido me colocó en una posición inestable como investigador, pues ponía en cuestión no solo el diseño inicial de la investigación, sino el propio sentido de investigar desde una perspectiva cualitativa. Me sucedía, como señala Green (2013), que los movimientos de la investigación se configuraban como

"a continual process of flux or differentiation even though this fact is usually masked by powerful and pervasive illusory discourses of fixity, stability, and identity that have characterized most of western philosophy and theory since at least Enlightenment"' (Green, 2013, p. 751).

La situación de investigación me demandaba una actitud investigadora nómada que implicaba imaginar una práctica de "traveling in the thinking that writing produces, in search of the field" (St. Pierre, 2000, p. 258). Un enfoque nómada, inestable y que requería una constante abertura ante lo imprevisto, que generaba relacionarme con las características perturbadoras en las que iba deviniendo la investigación. Algo similar sucedió cuando me encontré ante las cartografías y traté de mapear las trayectorias de los docentes, no como resultados, sino "questioning guidelines, the points of entry and exit, as a constant deployment" (Braidotti, 2006, p. 160). Esta operación que dejaba de ser normativa, supuso cuestionar, como señalan Snaza y Weaver (2014), el fenómeno del metodocentrismo, según el cual la metodología del investigador y su fidelidad a un método son la principal preocupación, relegando a los seres humanos, otros seres vivientes y objetos inanimados en una posición subordinada, desde la cual el rol de estos seres y su propia realidad se eliminan del trabajo del investigador.

Llegados a este punto, la pregunta inicial de la investigación ('Cómo el profesorado aprende dentro y fuera de su trabajo sobre: los contenidos disciplinares; los referentes pedagógicos; el aprender de los alumnos; las tecnologías, en particular las digitales; las dimensiones culturales y sociales; y los referentes de sí mismos’) se había transformado en otra cuestión que ahora no buscaba resultados, sino que se abría a un camino del pensar en los siguientes términos: 'Qué nos permite pensar, qué nos permite emerger el proceso de creación de cartografías por parte de profesores de secundaria en sus tránsitos de aprendizaje dentro y fuera de las instituciones educativas'. Este cambio nos llevó a nuevas cuestiones, que ya no buscaban respuestas, sino que se

\footnotetext{
9 “un proceso continuo de flujo o diferenciación a pesar de que este hecho es usualmente enmascarado por poderosos y penetrantes discursos ilusorios de fijación, estabilidad e identidad que han caracterizado la mayor parte de la filosofía y teoría occidental desde por lo menos la Ilustración" (Traducción del autor).
} 
abrían no sólo a otro sentido del aprender, sino de lo que puede significar investigar:

- ¿Cómo explorar "las sensaciones, intensidades y texturas a través de las cuales" (Coleman y Ringrose, 2014, p. 4) se experimenta el aprendizaje?

- ¿Cómo reinventar métodos de investigación para tratar la fluidez, la multiplicidad y la vaguedad (Law, 2004) de esas experiencias de aprendizaje?

- ¿Cómo comprender la multiplicidad de los mundos de aprendizaje de los docentes y las cuestiones que plantean los métodos que no sólo ‘captan' estas realidades, sino que las convierten en realidad?

- ¿Cómo trazar un mapa de las relaciones en las que los investigadores están siempre involucrados? (Hernández-Hernández, Sancho-Gil y Domingo-Coscollola, 2018).

Todo lo anterior se vincula a una noción de aprender que emerge en la investigación y que, como señala Atkinson, se caracteriza por ser un proceso creativo, que permite autorizarse, "que ha de tener momentos y formas de estabilización, ya que no siempre se puede estar avanzando. Tiene que haber puntos de estabilidad. Pero es complejo ese "cómo se mueve uno" en términos de ontogénesis, pues aprender no es solo cognición, sino que está integrado afectivamente" (Atkinson, 2015, p. 4).

Este recorrido, que aquí se presenta poniendo el énfasis en los movimientos de la investigación que afectaron al investigador, también permitió obtener una serie de resultados. No entendidos como algo que está ahí fuera y que el investigador 'rescata' mediante una serie de estrategias de obtención y análisis de datos. Sino como lo que la realización de la investigación ha posibilitado pensar sobre cómo aprenden los docentes:

Los docentes aprenden en una trama de relaciones, en la que se vinculan lo biográfico y lo corpóreo, además de lo cognitivo.

Los docentes aprenden transitando desde el 'afuera' a la vida diaria de la escuela. En particular en las relaciones con los colegas y los estudiantes.

Los docentes dan cuenta de una dimensión extensa de su aprendizaje en la vinculación que realizan con sus experiencias de vida de aprender.

Estas experiencias no pueden contemplarse de forma aislada, pues los docentes "asignan un territorio o espacio en sus cartografías, a los diferentes ámbitos en los que aprenden (familia, amigos, ocio, cultura, escuela...), al tiempo que explicitan múltiples intersecciones y tránsitos entre unos y otros" (Bosco, Alonso Cano y Miño Puigcercós, 2019, p. 87).

Finalmente, los aprendizajes "se constituyen como fenómenos materiales discursivos hechos de organismos, objetos, tecnologías, tiempos, espacios, situaciones, experiencias, valores y conceptos enredados” (Sancho-Gil y Correa-Gorospe, 2019, p. 134).

\section{Para seguir pensando sobre el sentido de investigar en educación}

No sólo el trabajo que comenzaste a escribir no es el mismo que en el que terminas escribiendo, sino que incluso el ser humano que eras cuando comenzaste el proyecto no es el mismo en el que te has convertido al final (Coetzee, en Seoane, 2019, p. 8).

Las aportaciones de Isabelle Stengers $(1997,2010)$-una investigadora que transita en la intersección de los estudios científicos, la historia de la ciencia y el posthumanismo - me acompañan ahora "a encontrar nuestro camino cuando el camino estaba oculto porque nos habíamos desviado 
de las sendas que nos habían dicho que siguiéramos" (Ahmed, 2017, p. 33), pues me permite fundamentar el sentido de investigar que trato de compartir en este artículo. Stengers sospecha de los campos que pretenden ser ciencia exhibiendo una multitud de objetos de utillaje (batas y espacios de laboratorio, gráficos, estadísticas, secuencia normativa de escritura, vocabulario técnico, etc.). Frente a quienes ponen el énfasis en el continente, ella considera que lo que hace de una ciencia 'ciencia', es que busca formas de dejar que su 'objeto' hable o se convierta en un sujeto. El 'qué' del estudio debe poder participar, para sorprender al investigador. Cuando un investigador decide de antemano qué se tendrá en cuenta y cómo se presentarán los 'resultados', la sorpresa es imposible. Todo lo que puede suceder es 'confirmación' o 'negación'. Cuando esto sucede el sistema está completamente cerrado.

Comparto entonces con Stengers la necesidad de una ciencia abierta, una ciencia que no tenga miedo de recordar la construcción cultural-política-histórica de la ciencia dentro de las redes humanistas; una ciencia que vaya más allá de la ciencia tal como la conocemos para ayudarnos a pensar el significado de las relaciones negadas en las que siempre estamos enredados. Estas relaciones involucran a humanos, animales, máquinas y cosas (Snaza, et al. 2014, p. 52).

Todo lo anterior tiene repercusiones para la investigación en educación, si se pretende tal y como lo hace Stephanie Springgay (2015), expandir los desplazamientos esbozados en este artículo. Springgay reclama, en primer lugar, mantener una sensación de movimiento, que se posiciona frente el empleo de 'métodos' preestablecidos, y en favor de procedimientos que surgen "in the midst of the research process" (p. 81). En segundo lugar, adoptar una construcción procesual, que considera la investigación no como rígida o limitada por un método, sino como una "ongoing construction" (p. 84) que sólo puede conducir "a approximate rigorous abstractions” (ídem). Lo que implica desafiar las nociones tradicionales de investigación centradas en la causalidad lineal. Finalmente, respondiendo a la invitación de Elizabeth St. Pierre (2013), Springgay pide a los investigadores educativos que consideren la idea de una investigación sin datos prestablecidos, fijos, concretos y aislados, pues la investigación que la perspectiva postcualitativa persigue es lo que es relacional y procesual.

Una investigación que se configura, no en una planificación que predetermina antes de realizarla, sino en el proceso de escritura, pues "[W]riting is thinking, writing is analysis, writing is indeed a seductive and tangled method of discovery" (Richardson y St. Pierre, 2005, p. 96, énfasis en original) y que permite explorar lo que no sabemos y nos sorprende. Al tiempo que nos posibilita nuevas formas de comprensión de aquello que despierta nuestra curiosidad y que nos abre otros modos de pensar y conocer. Lo que hace que investigar como aprender se configure como un dar cuenta de nuestros encuentros con aquello "que es difícil, que se resiste a ser comprendido por completo en el presente" generando lo que Sara Ahmed denomina 'conceptos sudorosos' (2017, p. 27), conceptos que "aportan cosas al mundo" (ídem, p. 28) y no sólo a la carrera de los investigadores.

\section{Agradecimientos}

El proyecto APREN-DO ha sido parcialmente financiado por el Ministerio de Economía, Empresa y Competitividad. EDU2015-70912-C2-1-R y EDU2015-70912-C2-2-R.

Grupo de investigación ESBRINA-Subjetividades, visualidades y entornos educativos contemporáneos (2017SGR 12489. http://esbrina.eu )

Red de excelencia REUNI+D- Red Universitaria de Investigación e Innovación Educativa. Cambios Sociales y Retos para la Educación en la Era Digital. EDU2015-68718-REDT.

http:/ / reunid.eu 


\section{Referencias}

Atkinson, D. (2011). Art, Equality and Learning. Pedagogies Against the State. Rotterdam/Boston/Taipei: Sense Publishers.

Atkinson, D. (2015). The adventure of pedagogy, learning and the not-known. Subjectivity, 8(1), 4356. https://doi.org(10.1057/sub.2014.22

Atkinson, D. (2018). Art, Disobedience and Ethics. The Adventure of Pedagogy. Cham, Switzerlan: Palgrave. https://doi.org/10.1007/978-3-319-62639-0

Ahmed, S. (2018). Vivir una vida feminista. Traducción María Engueix. Barcelona: Edicions Bellaterra.

Barquín, J. (1995). La investigación sobre el profesorado. Estado de la cuestión en España. Revista de Educación, 306, 7-65.

Bosco, A., Alonso Cano, C. y Miño Puigcercós, R. (2019). Geografías e historias de aprendizaje de docentes de secundaria. Intersecciones, tránsitos y zonas de no saber. Educatio Siglo XXI, 37(2), 67-92. http://dx.doi.org/10.6018/i/387021

Braidotti, R. (2006). Transpositions. On Nomadic Ethics. Cambridge: Polity Press.

Canales, C., Padilla-Petry, P. y Gutiérrez, L. (2019). El no-saber en las cartografías sobre nuestro aprender como investigadores: una mirada post-cualitativa. Educatio Siglo XXI, 37(2), 49-66. http://doi.org/10.6018/j/387011

Coleman, R. \& Ringrose, J. (2014). Deleuze and research methodologies. Edinburgh: Edinburgh University Press.

Charmaz, K. (2000). Grounded theory: Objectivist and constructivist methods. In N. Denzin \& Y. Lincoln (Eds.), Handbook of qualitative research (pp. 509-535). Thousand Oaks CA, USA: Sage.

Charmaz, K. (2006). Constructing Grounded Theory: A Practical Guide Through Qualitative Analysis. London, United Kingdom: Sage.

Darling-Hammond, L. \& Richardson, N. (2009). Teacher learning. What matters? Educational Leadership, February, 43-53.

Deleuze, G. y Guattari, F. (2006). ¿Qué es la filosofía? (Traducción Thomas Kauf). Barcelona: Anagrama.

Deleuze, G. y Guattari, F. (2015). Mil mesetas. Capitalismo y esquizofrenia (6a ed., Traducción José Vázquez Pérez con la colaboración de Umbelina Larraceleta). Valencia: Pre-Textos.

Fendler, R. (2015). Navigating the eventful space of learning: Mobilities, nomadism and other tactical maneuvers. Barcelona: University of Barcelona. Tesis no publicada.

Green, J.C. (2013). On rhizomes, lines of flight, mangles, and other assemblages. International Journal of Qualitative Studies in Education, 26(6), 749-758.

https://doi.org/10.1080/09518398.2013.788763

Guattari, F. (2000). Cartografías Esquizoanalíticas. Buenos Aires: Ediciones Manantial.

Guattari, F. y Rolnik, S. (2006). Micropolitica. Cartografías del deseo. Madrid: Traficantes de Sueños. Hernández, F. (1996-1997). ¿Cómo aprenden los docentes? Kikiriki. Cooperación educativa, 42-43, 120 127.

Hernández-Hernández, F. (Coord.) (2010). Aprender a ser en la escuela primaria. Barcelona: Octaedro. 
Hernández-Hernández, F., Sancho-Gil, J. \& Domingo-Coscollola, M. (2018). Cartographies as spaces of inquiry to explore of teacher's nomadic learning trajectories. Digital Education Review, 33, 105-119. https://doi.org/10.1344/der.2018.33.105-119

Hernández-Hernández, F. y Revelles Benavente, B. (2019). La perspectiva post-cualitativa en la investigación educativa: genealogía, movimientos, posibilidades y tensiones, Educatio Siglo XXI, 37(2), 21-48. http://doi.org/10.6018/j/387001

Hernández-Hernández, F., Correa Gorospe, J.M., Aberasturi Apraiz, E. Sancho Gil, J.M. (Eds.) (2019). ¿Cómo aprenden los docentes? Aprender como movimiento cartográfico, performativo, experiencial y afectivo. Barcelona: Octaedro.

Hustvedt, S. (2019). La mujer que mira a los Hombres que miran a las mujeres, Ensayos sobre feminismo, arte y ciencia. Traducción Aurora Echevarría. Barcelona: Planeta.

Jackson, A. \& Mazzei, L. (2012). Thinking with theory in qualitative research: Viewing data across multiple perspectives. New York: Routledge.

Lather, P. (2013). Methodology-21: what do we do in the afterward? International Journal of Qualitative Studies in Education, 26(6), 634-645. https://doi.org/10.1080/09518398.2013.788753

Lather, P. \& St. Pierre, E. (2013). Post-qualitative research. International Journal of Qualitative Studies in Education, 26(6), 629-633. https://doi.org/10.1080/09518398.2013.788752

Law, J. (2004). After Method: Mess in Social Science Research. London \& New York: Routledge.

Marcelo, C. (1995). Constantes y desafíos actuales de la profesión docente. Revista de Educación, 306, 205-241.

Masny, D. (2013) (Ed.). Cartographies of Becoming in Education. A Deleure-Guattari Perspective. Rotterdam: Sense Publishers.

Opfer, D. V. \& Pedder, D. (2011). Conceptualizing Teacher Professional Learning. Review of Educational Research, 81(3), 276-407.

https://doi.org/10.3102/0034654311413609

Rancière, J. (2010). El espectador emancipado. Buenos Aires: Manantial

Richardson, L. \& St. Pierre, E. (2005). Writing. A method of Inquiry. In N. K. Denzin \& Y. S. Lincoln (Eds.), The SAGE Handbook of Qualitative Research (3a ed., pp. 959-978). Londres: SAGE Publications.

Sancho, J.M. y Hernández, F. (2015). Pedagogía de lo desconocido. Una entrevista a Dennis Atkinson. Cuadernos de Pedagogía, 454, 34-38.

Sancho-Gil, J. M. y Correa-Gorospe, J.M. (2019). Intra-acciones en el aprender de docentes de infantil, primaria y secundaria. Educatio Siglo XXI, 37(2), 115-140. http://dx.doi.org/10.6018/i/387041.

Semetsky, I. (2006). Deleuze, Education and Becoming. Rotterdam; Taipei: Sense Publishers.

Seoane, A. (2019). J.M. Coetzee "Ni yo deseo comprender plenamente que escribo". El Cultural, 21 de junio, 8-10.

Shulman, L. S. \& Shulman, J. H. (2004). How and What Teachers Learn: A Shifting Perspective. Journal of Curriculum Studies, 36(2), 257-271. https://doi.org/10.1080/0022027032000148298 
Snaza, N., Appelbaum, P., Bayne, S., Carlson, D., Morris, M., Rotas, N., Sandlin, J., Wallin, J. \& Weaver, J. (2014). Toward a Posthumanist Education, Journal of Curriculum Theorizing, 30(2), 39-55.

Snaza, N. \& Weaver, J. (Ed.). (2015). Posthumanism and Educational Research. New York, NY: Routledge.

Springgay, S. (2015). Approximate-Rigorous-Abstractions": Propositions of Activation for Posthumanist Research. In N . Snaza \& J. Weaver (Ed.). Posthumanism and Educational Research. (pp.76-88). New York, NY: Routledge.

Stengers, I. (1997). Power and invention: Situating science. Minneapolis, MN: University of Minnesota Press.

Stengers, I. (2010). Cosmopolitics I. Minneapolis, MN: University of Minnesota Press.

St. Pierre, E. (2011). Post Qualitative Research. The Critique and Coming After. In N. Denzin \& Y.S. Lincoln (Eds.). The SAGE Handbook of Qualitative Research. (3rd ed.). (p. 611-625). Thousand Oaks, CA: SAGE.

Ulmer, J. B. \& Koro-Ljungberg, M. (2015). Writing Visually Through (Methodological) Events and Cartography. Qualitative Inquiry, 21(2), 138-152.

\section{Información sobre el autor}

Autor: Fernando Hernández-Hernández

Institución: Universidad de Barcelona

Email: fdohernandez@ub.edu

Grupo de investigación Esbrina: http://esbrina.eu

ORCID: https://orcid.org/ 0000-0003-0375-1830 


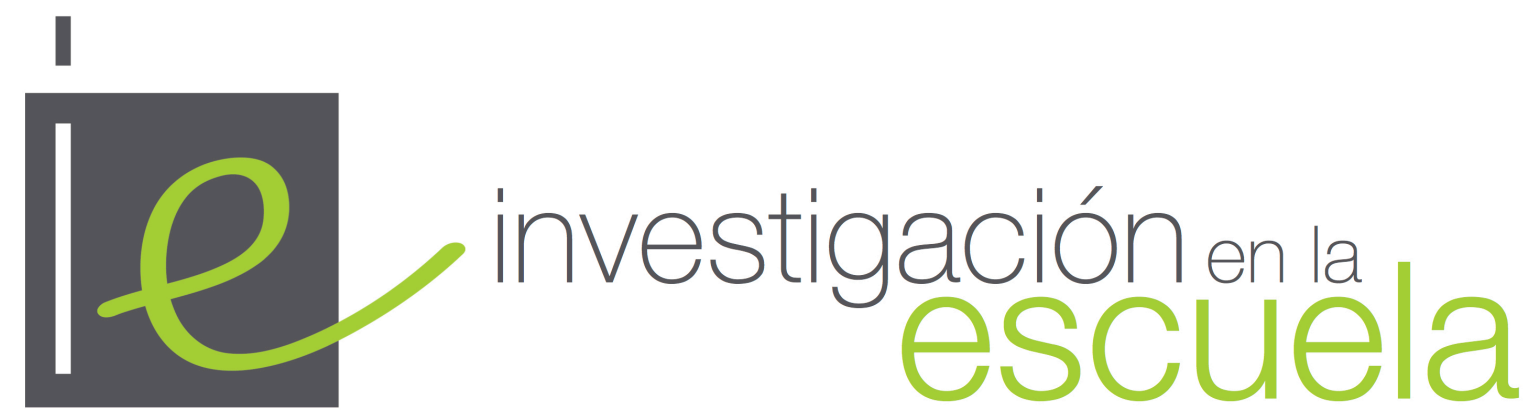

Revista académica evaluada por pares y de acceso abierto

Número 99

30 de diciembre de 2019

ISSN 2443-9991

\section{c) (i) (2)}

Esta obra está bajo una licencia Creative Commons. Los/as lectores/as pueden compartir, copiar y redistribuir el material en cualquier medio o formato, así como adaptar, remezclar, transformar y construir a partir del material para cualquier propósito, incluso comercialmente. Para ello, deben de hacerlo bajo los siguientes términos: dando crédito de forma adecuada, brindando un enlace a la licencia e indicando si se han realizado cambios. Si se remezcla, transforma o crea a partir del material, debe distribuir su contribución bajo la misma licencia del original.

Más detalles de la licencia de CreativeCommons se encuentran en https://creativecommons.org/licenses/by-sa/4.0/deed.es

Cualquier otro uso debe ser aprobado en conjunto por el autor/es, o Investigación en la Escuela.

ư

Revista Editada por la Universidad de Sevilla. https://editorial.us.es/es/revistainvestigacion-en-la-escuela

Por errores y sugerencias contacte a secretaria@investigacionenlaescuela.es

La revista Investigación en la Escuela desde su origen en 1987 hasta su nº 87 (2015) fue editada por Díada Editora. 
